\title{
LOW -ENERGY ADAPTIVE CLUSTERING HIERARCHY USING VIRTUAL GIRD METHOD
}

\author{
Meenakshi Yadav \\ CSE, AIM \& ACT \\ Banasthali University \\ Tonk, Rajasthan, India
}

\author{
Anoop Bhola \\ CSE, AIM \& ACT \\ Banasthali University \\ Tonk, Rajasthan, India
}

\author{
C.K.Jha \\ CSE, AIM \& ACT \\ Banasthali University \\ Tonk, Rajasthan, India
}

\begin{abstract}
It is expected from the wireless network to run continuously for long duration. For increasing network life there are various energy efficient techniques are available like cluster architectures, Energy as routing metrics, multipath routing ,Relay node placement and sink mobility. Clustering is one of the main techniques to improve the network life. There are various clustering protocol were introduced in past couple of years, like LEACH, LEACH-B,TEEN, APTEEN etc. In this paper we propose a new clustering protocol LEACH-VGM, which is using clustering technique and virtual gird method. Virtual gird method is used to divide the network in clusters. LEACH has used the probability to select cluster head but it does not consider remaining energy of node. Simulation results shows using of virtual gird method improved the network life.
\end{abstract}

Keywords: LEACH, Virtual Gird, Cluster Head, Clustering, Base Station

\section{INTRODUCTION}

In recent years, sensors used Micro Electro Mechanical System (MEMS ) technology. Sensors are very small in size and having limited resources like energy. A wireless sensor network has number of sensors for sensing the fields and that information gathered at base station. Generic and gateway are the two sensor types. Generic is a multipurpose sensor nodes those take measurements form the monitor environment. It may equip with Varity of devices. Gateway nodes gathered data from generic nodes and relay them to the base station. Jennifer Yick [6], focuses on recent literature and gives the issues related to individual and group sensor nodes in wireless sensor network. Author has covered the issues like localization; coverage of various wireless sensor network. Finally, author provides the summary of current sensors technologies.

TiffennRault [7], gives a top-down survey of designing wireless sensor network in terms of application requirement and lifetime extension. The author has provided the categories of applications and their specific requirements. Also, classification of energy saving schemes and their specific requirements has been discussed. Main requirements identified by the author are scalability, coverage, latency, QoS, Security, mobility and robustness. Finally author provides a classification of energy-efficient mechanisms.

For improving the scalability clustering technique has been used in many protocols of wireless sensor network. LEACH is first protocol that introduces clustering technique for improving the network life. LEACH has used probability theory for selecting cluster head. Cluster head is basically a gateway for the cluster. It collects the data and sends it to base station. Dividing the deployments area in clusters is also important.

In this paper we use virtual gird method to divide the network into clusters. These clusters fulfill the criteria portability of cluster head selection which is not available in LEACH.

The rest of the paper organized as follows. In the next section various protocols those are related with LEACH are explained. Section 3 explained the virtual grid method and proposed algorithm. Simulation results are given in section 4 . Section 5 concludes the paper.

\section{LITERATURE SURVEY}

Paul Lettieri et al [1], presents a problem description regarding data movement in wireless sensor networks. For the illustration, they have presented the architecture with two features; 1) Intra-node router and 2) functional repartitioning. They claim that their architecture reduces traffic through router.

Sandeep Tanwar [3], compares various heterogeneous routing protocols for wireless sensor network with predefined parameters like $\mathrm{CH}$ selection, energy efficiency and application. For analysing performance of the wireless sensor network various parameters are listed by the author like: type of deployment of sensor nodes, the lifetime of the network field, latency, packet delivery ratio, connectivity, scalability, reliability, stability, energy efficiency and minimization of cost of energy consumption. Author also states that heterogeneity is playing major role in network life. It decreases the latency and increases the throughput of data transfer from source to destination.

Maryam Sabet [4], proposed a clustering approach called MLRC (Multi-Level Route aware Clustering). In MLRC, nodes can have desired information of possible route to the destination. This approach also clears extra generation of 
routing control packets by implementing cluster formation and routing tree construction.

Ado Adamou [5],proposed a routing algorithm called ABCSD using artificial Bee Colony approach and designed a multiobjective fitness function for cluster head selection. The authors have used the cost based function to solve the routing problem.

Meenakshi Sharma [8], author has proposed a multilevel clustering technique. They have introducedanothercluster layer between nodes and the base station. Second layer is having a master cluster head for decreasing the communication distance. Number of clusters and communication distance are inversely proportional to each other. Master cluster head receives data from nearest cluster heads and sent it to the base station. This proposed technique reduces the control overhead on CHs and equally distribute the load. Author also gives the distance calculation formula. But after a certain time $\mathrm{MCH}$ also drain their energy and then situation is similar to LEACH. Ravi Kishore [9], proposes a new protocol which is based on LEACH. Hierarchical approach has been used. Name of those techniques are 2L-LEACH, 3L-LEACH, 4L-LEACH. Selection of these techniques are based on Network Size, Larger size will opt maximum level. Simulation results show improvement in LEACH using level technique.

SapanaGambhir [10],proposed a new protocol called OPLEACH (optimized). Performance has been measured via time delay and energy. It uses slots those are free and they have no data to send. These slots have been used for TDMA schedule, so that frames get extra space for data communication. Data transmission delay, waiting time has been reduced and throughput of the network has been increased.

Mahmoud M. Salim [11], proposed a new technique for improvement in LEACH called PR-LEACH. PR-LEACH has distributed the energy load to sensor nodes. Base station calculates the energy range using the information of residual energy provided by the cluster head. By this,the information is finalized. Author also proposed a threshold formula.

Shipla Mahajan [12], has used graph theory concept to find the minimum distance from source to destination. BFS technique has been used to divide the network into sub regions and calculate the energy at each phase.

Kamaljhot [13], describes the various protocols those are more efficient than LEACH. A comparison table shows the efficiency of those protocols. Details of various LEACH extension protocol are also given.

HossienSalmabadi [14], divides the area into zones. For calculating the number of zones,equations have been used. Because the threshold value has been calculated according to the zone, thus no advertisement message is needed. Cluster head has been selected according to the distance with respect to the base station.

Mohammed Omari [15],analysed and simulated different clustering protocol like LEACH, LEACH-C, LEACH-1R, and HEED. Comparison is based on residual energy, delivery of data to base station, number of rounds and live nodes.

SamanSiavashi [16], introduces a new clustering protocol for load balancing. In the protocol network is divided into virtual circle having variable radiuses. This radiuses and cluster size will increase with respect to distance to the base station. Leader nodes are used for collecting and compressing the data load balancing and balanced distribution of energy. Consumption around the network is also achieved by this protocol.
Heydar [17], author has used Bayesian Network model to select cluster head. Bayesian network model is based on 3 factor, distance to base station, energy remaining and density. This model is also used to calculate probability to become a cluster head for each sensor node. The field is divided into three regions, far, central and near. Central region have highest prior probability, near having medium and far have lowest. Jong-Yong Lee [18], proposed DL-LEACH protocol to solve rapid energy consumption. Author also changed the transmission method of LEACH single to dual hop. Using dynamic clustering and partial single-hop transmission energy consumption has been improved.

WassimJerbi [19], proposed O-LEACH (orphan) protocol. This protocol has reduced the sensor nodes which are not related to cluster. In O-LEACH two main parts are introduced. In first part, orphan nodes joined to the gateway node. Gateway node is the member node of cluster. So gateway node is behaving like cluster head for orphan nodes. In second part, the number of nodes is greater than member nodes of cluster a sub cluster will be created. O-LEACH achieves long life and minimum energy consumption.

Anup Bhola [21] ,mainly consider the balanced the unequal clustering technique and gives the new distance calculation formula .Anup Bhola [22], proposed the new handshaking technique for communicating the cluster heads and nodes which are not in cluster area.

\section{VIRTUAL GIRD METHOD}

The VGM method divides the network into similar sized clusters. Reason behind this is to divide the load similarly in cluster head. Cluster head are directly related to the network life. They play important role in network life. For optimal cluster head we used the same method that used in LEACH [2], which consider $5 \%$ of total nodes.[20]

Equation 1 is used to divide the network. If number of sensor node is 100 total number of cluster is 4 . And if total number of sensor nodes is 200 clusters are 6 and so on. Figure 1,2and 3 shows the network division.

$$
\mathrm{K}= \begin{cases}4 & \mathrm{~N} * 0.05<=6 \\ 9 & 6<\mathrm{N} * 0.05<=12 \\ 16 & 12<\mathrm{N} * 0.05<=20\end{cases}
$$

Following are the main important advantages of dividing the network

- $\quad$ Cluster head distribution: Cluster head distribution is based on heuristics that is $5 \%$ cluster head of total number of nodes.

- Number of cluster heads is same in all rounds and $\mathrm{CH}$ is selected according to number of zones.

Algorithm

- Formation of sensor network

- Initialize $\mathbf{N}=$ Number of sensor nodes

- $\quad$ Sink $=0.05^{*} \mathrm{x}$ and $0.05^{*} \mathrm{y} / *^{*}$ Initialize Sink coordinates */

- Calculate the number of zones according to number of nodes using equation $\mathbf{1}$

- If $\mathbf{k}<=6$

- Then

- Number of zones are $\mathbf{4}$

- For $\mathbf{i}$ to $\mathbf{N}$

- Deploy the sensor node randomly in an area 
- $\quad$ CH selection according to LEACH algorithm

- Else if $\mathbf{K}<=12$

- Number of zones are $\mathbf{9}$

- $\mathbf{C H}$ selection according to LEACH algorithm

- Else

- Number of zones are $\mathbf{1 6}$

- $\mathbf{C H}$ selection according to LEACH algorithm

\section{Simulation Results}

In this section, we present the simulation results using MATLAB. We took 100 sensor nodes deployed randomly in $100 X 100$ areas. Initially every node has 0.1J energy. We consider the energy model used in [2]. We used $\mathrm{E}_{\text {elec }}, \mathrm{E}_{\mathrm{Tx}}, \mathrm{E}_{\mathrm{Rx}}$ $=50 \mathrm{~nJ}$, Eamp $=13 \mathrm{pJ} / \mathrm{bit} / \mathrm{m}^{2}, \mathrm{Efs}=10 \mathrm{~nJ} / \mathrm{bit} / \mathrm{m}^{2}$. Packet length is 6400 bit. Figure 4 shows the dead nodes of LEACH and LEACH-VGM. First node died at round 42 in LEACH-VGM and for LEACH it's $32^{\text {nd }}$ round. After completing 100 round the ratio is 23:42 for died nodes, where 23 for LEACH-VGM and 42 for LEACH. When LEACH first node die the number of died node increase at each round, but on LEACH-VGM it remains stable till $62^{\text {nd }}$ round shows in figure 4 .

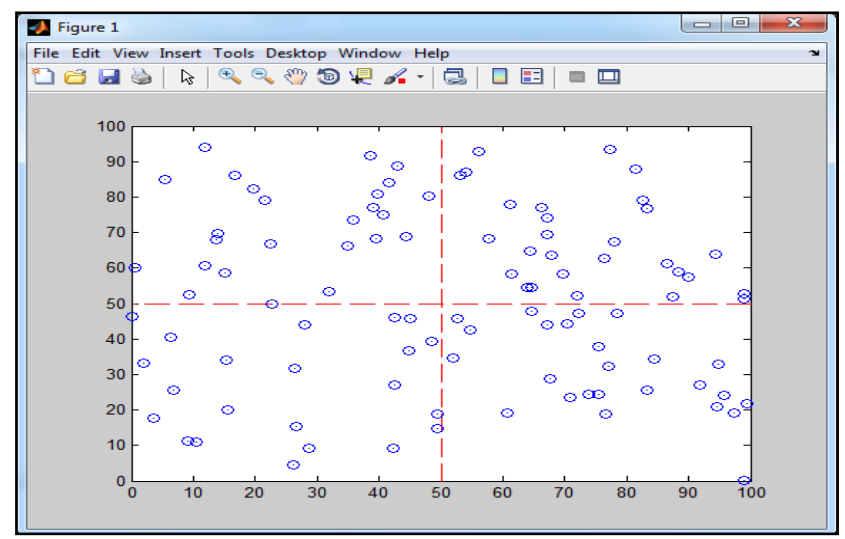

$\mathrm{N}=100$ nodes deployment

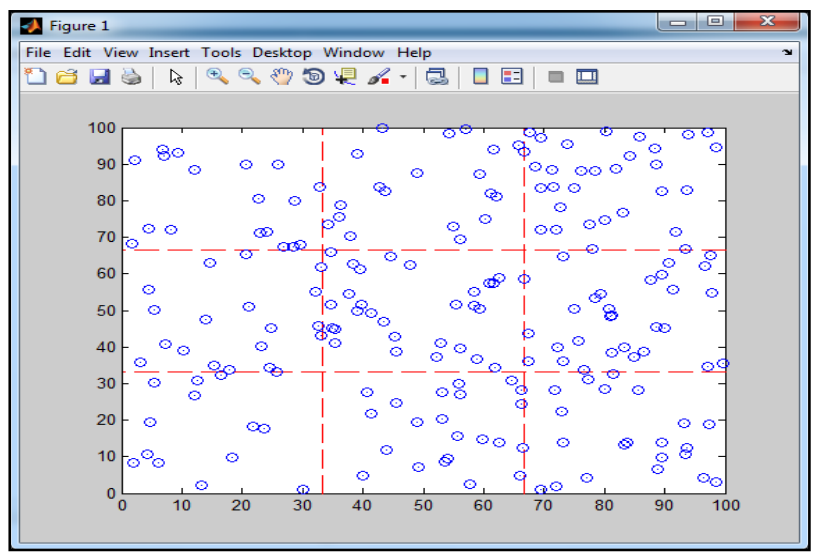

Fig.2. $\mathrm{N}=200$ nodes deployment

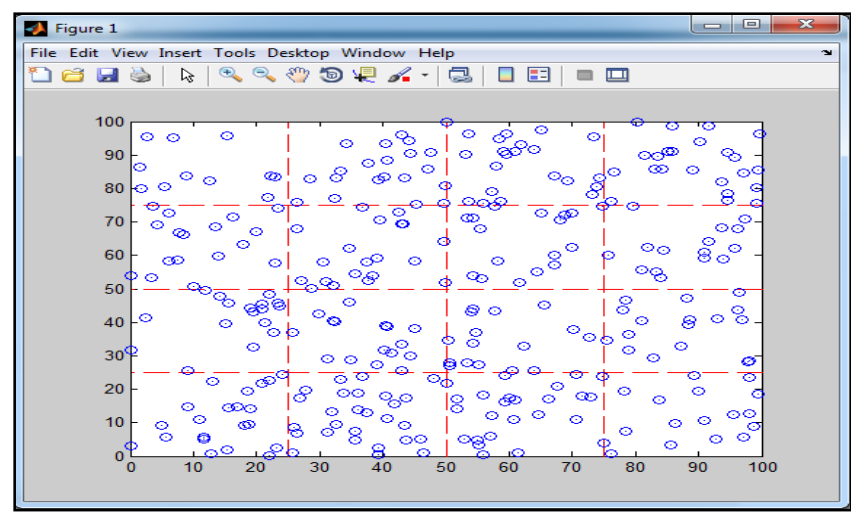

Fig.3.N=300 nodes deployment

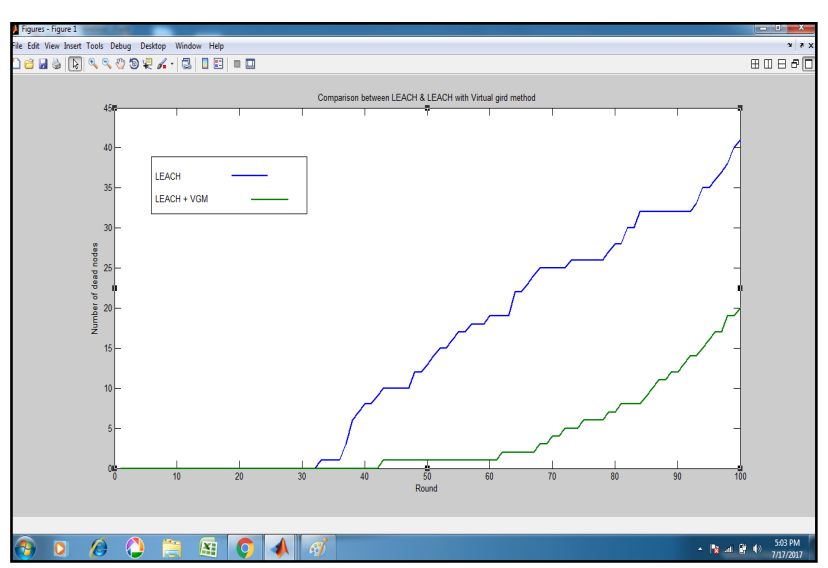

Fig. 4. LEACH vs LEACH-VGM dead nodes

\section{CONCLUSION}

Using virtual gird method LEACH-VGM evenly selects the cluster head in network and perfectly worked on the concept of $5 \%$ cluster head for 100 nodes. Simulation results show the deployment of sensor nodes in network using LEACH-VGM .LEACH-VGM improves the network life.

\section{REFERENCES}

[1] Paul Lettieri and Mani B. Srivastava: A QoS-aware, Energy-efficient Wireless Node Architecture: IEEE international workshop on mobile multimedia communication, pp. 252-261, 1999.

[2] Wendi Rabiner Heinzeleman, Anantha Chandrakasan and Hari Balakrishnan:Enegry Efficient communication protocol for wireless microsensor networks: proceeding of the IEEE 33rd international conference on System Sciences 2000.

[3] Sundeep Tanwar, Neeraj Kumar, Joel J.P.C. Radrigues: A systematic review on heterogeneous routing protocols for wireless sensor network : In Journal of Network and Computer application 53(2015)39-56. 2015.

[4] Maryam Sabet, Hamidreza Naji: An energy efficient multi-level route-aware clustering algorithm for wireless sensor networks: Computer Science and Electrical Engineering ELSEVIER 000(2016) 1-19, August 2016.

[5] Ado Adamou Abba Ari, Blaise Omer Yenke, Nabila Labraoui, Irepran Damakoa, Abdelhak Gueroui: “A power efficient cluster-based routing algorithm for wireless sensor networks: Honeybees swarm intelligence based 
approach":Network and Computer Applications ELSEVIER 69(2016 77-97, April 2016.

[6] Jennifer Yick, Biswananth Mukherjee, Dipak Ghosal: Wireless sensor network survey: computer networks ELSEVIER 52(2008)2292-2330, April 2008.

[7] Tifenn Rault, Abdelmadjid Bouabdallah, Yacine Challal: Energy efficiency in wireless sensor networks: A topdown survey: Computer Networks ELSEVIER 67(2014) 104-122, April 2014.

[8] Meenakshi Sharma, Kalpana Sharma: An Energy Efficient Extended LEACH (EEE LEACH): IEEE international conference on communication systems and network technologies 2012. DOI 10.1109/CSNT.2012.88.

[9] Ravi kumar kodali, Naveen kumar Aravapalli: Multi-level LEACH protocol model using ns-3: IEEE international Advance Computing conference (IACC) 2014. 978-14799-2572-8/14.

[10] Sapna Gambhir, Nida Fatima: Op-LEACH : An optimized LEACH method for busy traffic in WSNs: IEEE 4th international conference on Advanced computing \& communication Technologies 2014, 978-1-4799-49106/14.

[11] Mohmoud M. Salim, Hussein A. Elsayed, Salwa H. El Ramly: PR-LEACH: approach for Balancing Energy Dissipation of LEACH protocol for Wireless Sensor Networks: IEEE 31st National Radio Science Conference (NRSC2014) Egypt April 2014, 978-1-4799-3821-6/14.

[12] Shipla Mahajan, Jyoteesh Malhotra, Sandeep Sharma: Energy balanced optimum path determination based on graph theory for wireless sensor network: IET wireless sensor systems 2015, vol. 5, ISS. 6,pp 290-298, August 2015.

[13] Kamaljyot Singh : WSN LEACH based protocols: A structural Analysis: IEEE international conference and workshop on computing and communication (IEMCON) , Oct 2015, 978-1-4799-6908-1/15.

[14] Hassein Salmabadi, Mehdi Agha Sarram , Fazlollah Adibnia: An Improvement on LEACH protocol (EZLEACH): IEEE 2nd international conference on knowledge based engineering and innovation. November 2015. 978-1-4673-6506-2/15.
[15] Mohammed omari, Warda Hadj Fateh: Simulation comparison and Analysis of wireless sensor networks protocols: LEACH, LEACH-c, LEACH-1R and HEED: IEEE 4th international conference on Electrical Engineering, Dec 2015, 978-1-4673-6673-1/15.

[16] Saman Siavoshi, Yousef S. Kavian, Hamid Sharif: Load Balanced energy efficient clustering protocol for wireless sensor networks: IET wireless sensor systems 2016, vol. 6 , ISS. 3, pp. 67-73.

[17] Heydar Ghasemzadeh, Mehdi Rezaeian, Fatemeh Dehghan, Mohammad Mohsen: BN-LEACH : An Improvement on LEACH protocol using Bayesian Networks for energy consumption reduction in wireless sensor networks: IEEE 7th international symposium on telecommunications (IST-2014), 978-1-4799-5359-2/14.

[18] Jong-yonglee, kye-Dong Jung, Scok0Jarmoon, Hwayoung Jeang: Improvement on LEACH protocol of a wide area wireless sensor networks: multimedia tools application SPRINGER DOI 10.1007/s11042-016-37324, july 2016.

[19] Wassim Jerbi, Abderrahmen Huermazi, Hafedh Trabelsi: O-LEACH of routing protocol for wireless sensor networks: IEEE 13th international conference computer graphics, Imaging and Visualization 2016. 978-1-50900811-7/2016.

[20] Abdul Waheed Khan, Abdul Hanan Abdullah: VGDRA: A Virtual Grid-Based Dynamic Routes Adjustment Scheme for Mobile Sink-Based Wireless Sensor Networks: IEEE Sensor Journal vol 15. No. 1 January 2015. DOI 10.1109/JSEM.2014.2347137.

[21] Anup Bhola, Mallika Mhatre "Energy efficient wireless sensor's routing using balanced unequal clustering technique" presented in First International Conference on Smart Technologies in Computer and Communication (SmartTech-2017)

[22] Anup Bhola, Mallika Mhatre ,CK Jha "Energy efficient WSN using membership handshaking clustering technique for isolated nodes" presented in First International Conference on Smart Technologies in Computer and Communication (SmartTech-2017) 